ARTICLE

\title{
Robust metal ion-chelated polymer interfacial layer for ultraflexible non-fullerene organic solar cells
}

Fei Qin (1) 1,5, Wen Wang (1) 1,5, Lulu Sun (1) 1,5, Xueshi Jiang (1) 1, Lin Hu (1) 1, Sixing Xiong (1) 1, Tiefeng Liu (1) 1, Xinyun Dong (1) ${ }^{1}$, Jing Li (i] 1, Youyu Jiang', Jianhui Hou (1) ${ }^{2}$, Kenjiro Fukuda ${ }^{3}$, Takao Someya (1) ${ }^{3,4}$ \& Yinhua Zhou (1) ${ }^{1 \times}$

Achieving high power conversion efficiency and good mechanical robustness is still challenging for the ultraflexible organic solar cells. Interlayers simultaneously having good mechanical robustness and good chemical compatibility with the active layer are highly desirable. In this work, we present an interlayer of $\mathrm{Zn}^{2+}$-chelated polyethylenimine (denoted as $\mathrm{PEI}-\mathrm{Zn}$ ), which can endure a maximum bending strain over twice as high as that of $\mathrm{ZnO}$ and is chemically compatible with the recently emerging efficient nonfullerene active layers. On $1.3 \mu \mathrm{m}$ polyethylene naphthalate substrates, ultraflexible nonfullerene solar cells with the PEI-Zn interlayer display a power conversion efficiency of $12.3 \%$ on PEDOT:PSS electrodes and $15.0 \%$ on AgNWs electrodes. Furthermore, the ultraflexible cells show nearly unchanged power conversion efficiency during 100 continuous compression-flat deformation cycles with a compression ratio of $45 \%$. At the end, the ultraflexible cell is demonstrated to be attached onto the finger joint and displays reversible current output during the finger bendingspreading.

\footnotetext{
${ }^{1}$ Wuhan National Laboratory for Optoelectronics, Huazhong University of Science and Technology, Wuhan 430074, China. ${ }^{2}$ Beijing National Laboratory for Molecular Sciences, State Key Laboratory of Polymer Physics and Chemistry, Institute of Chemistry, Chinese Academy of Sciences, 100190 Beijing, China. ${ }^{3}$ Thin-Film Device Laboratory \& Center for Emergent Matter Science (CEMS), RIKEN, 2-1 Hirosawa, Wako, Saitama 351-0198, Japan. ${ }^{4}$ Department of Electrical Engineering and Information Systems, The University of Tokyo, 7-3-1 Hongo, Bunkyo-ku, Tokyo 113-8656, Japan. ${ }^{5}$ These authors contributed equally: Fei Qin, Wen Wang, Lulu Sun. ${ }_{\text {email: yh_zhou@hust.edu.cn }}$
} 
O rganic solar cells (OSC) have been attracting great attentions due to their easy processing and increasing power conversion efficiency (PCE) ${ }^{1-11}$. Comparing to inorganic counterparts, the organic and polymer semiconductors have excellent mechanical flexibility which enables the fabrication of ultraflexible and stretchable organic optoelectronic devices ${ }^{12-14}$. The ultraflexible OSCs are attractive for the integrated applications in intelligent robots, electronic skin, and Internet of Things ${ }^{15-22}$. In 2011, the Bao group reported the stretchable OSCs on pre-stretched polydimethylsiloxane (PDMS) with poly(3-hexylthiophene):phenyl-C61-butyric acid methyl ester (P3HT:PCBM) active layer, obtaining a PCE of $2.0 \%{ }^{23}$. Later, ultraflexible OSCs are reported on ultrathin polymer substrates (such as polyethylene terephthalate (PET), parylene, etc). The ultrathin OSCs can be transferred onto the pre-stretched elastomer to realize the stretchability. For example, Kaltenbrunner et al. reported an ultrathin OSC fabricated on 1.4- $\mu \mathrm{m}$-thick PET and achieved a PCE of $4.2 \%{ }^{24}$. Recently, the Someya group fabricated the ultrathin solar cells sandwiched between two parylene substrates ${ }^{25,26}$. Their PCE reaches about $10 \%$ and the cells show good thermal stability and water-proof properties.

Although the attractive features of the ultraflexible solar cells have been demonstrated, mechanical durability of the cells during the continuous deformation cycling at a micrometer-scale bending radius is still challenging. To obtain good mechanical robustness, all the functional layers (including electrodes, interlayers, and the active layer) are required to have good mechanical flexibility. The active layer is based on polymer-based mixture that has good mechanical flexibility. Poly(3,4-ethylenedioxythiophene): polystyrene sulfonate (PEDOT:PSS) and silver nanowires (AgNWs) electrodes are good options as flexible electrodes. Thus, proper design of the interlayers and electrodes is important. Typically used $\mathrm{ZnO}$ interlayer tends to crack under high bending strain that is detrimental to the mechanical robustness of the ultraflexible $\mathrm{OSCs}^{27}$. A mechanically robust interlayer is needed to achieve good deformation cycling durability. Amorphous polymer-based interlayer could meet the request due to their good mechanical flexibility ${ }^{27,28}$. Aminecontaining polymer polyethylenimine (PEI) has been used to produce electron-collecting interface in fullerene solar cells ${ }^{29}$. But, high-performance nonfullerene acceptors tend to react with PEI. The reaction destroys their chemical and electronic structure of the acceptor ${ }^{30,31}$ and results in poor performance. Previously, we proposed a protonation strategy to suppress the reaction by changing the processing solvent from alcohol to water for PEIE ${ }^{31}$. This strategy only works well with the indium tin oxide (ITO) electrode which has poor mechanical robustness, but not with the flexible PEDOT:PSS electrode or AgNWs electrode. Protonated PEIE is an insulator and its thickness should be thin (about 10 $\mathrm{nm}$ ) to ensure efficient electron extraction. It cannot be used to smoothen the rough surface of AgNWs. Therefore, it is important to develop interlayers that can endure high mechanical strain and be simultaneously compatible with the nonfullerene active layers and flexible electrode to fabricate ultraflexible OSCs with high PCE and good mechanical robustness.

In this work, we present a robust low-work function interlayer of Zinc ion $\left(\mathrm{Zn}^{2+}\right)$ chelated PEI (denoted as PEI-Zn) for efficient ultraflexible nonfullerene OSCs. The PEI-Zn can endure a maximum bending strain over twice as high as that of $\mathrm{ZnO}$. Simultaneously, the chelation of $\mathrm{Zn}^{2+}$ with the PEI reduces the chemical reactivity of PEI, and therefore the reaction between the PEI and the nonfullerene active layer is suppressed. The PEI-Zn can work efficiently as the interlayer with the flexible electrodes of PEDOT:PSS and AgNWs to realize ultraflexible OSCs. With the PEI-Zn, ultraflexible OSCs show a PCE of $12.3 \%$ with PEDOT: PSS electrode and a PCE of $15.0 \%$ with AgNWs. The cells show good mechanical durability during the continuous deformation cycling with a compression ratio of $45 \%$.

\section{Results}

Structure and photovoltaic performance of ultraflexible OSCs. Figure 1 shows the device structure of the inverted ultrathin OSCs (PEN/PEDOT:PSS or AgNWs/PEI-Zn/active layer/ $\mathrm{MoO}_{3} / \mathrm{Ag}$ ). $1.3-\mu \mathrm{m}$-thick polyethylene naphthalate $(\mathrm{PEN})$ is used as the substrate. PEDOT:PSS and AgNWs is used as the bottom transparent electrode. Two nonfullerene active layers of PBDB-T-2F: IT- $4 \mathrm{~F}^{32,33}$ and PBDB-T-2F:Y6 $6^{34}$ are tried in the ultraflexible OSCs. Their chemical structures are shown in Fig. 1a. PEI-Zn interlayer is deposited between the bottom flexible electrode and the nonfullerene active layer for electron collection. The PEI-Zn is the key part of this work. Design and properties of PEI-Zn will be included in the next section. Pictures of the fabricated ultrathin nonfullerene solar cells are shown in Fig. $1 \mathrm{~b}$.

Figures 1c, d shows the current density-voltage $(J-V)$ characteristics of the four different types of ultrathin OSCs: with active layer PBDB-T-2F:IT-4F or PBDB-T-2F:Y6 on PEDOT:PSS or AgNWs electrodes, respectively. When PBDB-T-2F:IT-4F is used as the active layer (Fig. 1c), cells with PEDOT:PSS electrode show a PCE of $10.4 \%\left(V_{\mathrm{OC}}=0.83 \mathrm{~V}, J_{\mathrm{SC}}=17.4 \mathrm{~mA} \mathrm{~cm}^{-2}, \mathrm{FF}=\right.$ 0.72 ) and cells with AgNWs electrode show a PCE of $12.9 \%$ $\left(V_{\mathrm{OC}}=0.82 \mathrm{~V}, J_{\mathrm{SC}}=21.0 \mathrm{~mA} \mathrm{~cm}^{-2}, \mathrm{FF}=0.75\right)$. When PBDBT-2F:Y6 is used as the active layer (Fig. 1d), cells with PEDOT: PSS electrode show a PCE of $12.3 \% \quad\left(V_{\mathrm{OC}}=0.83 \mathrm{~V}, J_{\mathrm{SC}}=\right.$ $\left.21.2 \mathrm{~mA} \mathrm{~cm}^{-2}, \mathrm{FF}=0.70\right)$ and cells with AgNWs electrode show a PCE of $15.0 \%\left(V_{\mathrm{OC}}=0.83 \mathrm{~V}, J_{\mathrm{SC}}=25.4 \mathrm{~mA} \mathrm{~cm}^{-2}, \mathrm{FF}=0.71\right)$. The cells on the AgNWs electrode (sheet resistance of $30 \mathrm{ohm} \mathrm{sq}{ }^{-1}$ ) show higher $J_{\mathrm{SC}}$ than those on PEDOT:PSS electrode (sheet resistance of $110 \mathrm{ohm} \mathrm{sq}^{-1}$ ) due to the lower transmittance of the PEDOT:PSS film (Supplementary Fig. 1, the transmittance spectra measured with an integrating sphere). This is consistent with the external quantum efficiency (EQE) results where lower EQE values were observed in the long-wavelength region for cells with PEDOT:PSS electrodes (Supplementary Fig. 2). The output current remains $98 \%$ of the initial current after $6 \mathrm{~h}$ continuous illumination (Supplementary Fig. 3). PCE distribution histograms of individual cells with different electrodes and active layers are displayed in Supplementary Fig. 4. The device stability in air (with $100-\mathrm{nm} \mathrm{Al}_{2} \mathrm{O}_{3}$ encapsulation by atomic layer deposition, relative humility: 15\%) is also tested. The devices remain over $80 \%$ of its initial efficiency under $200-\mathrm{h}$ continuous illumination (Supplementary Fig. 5). There is no hysteresis observed in the $J-V$ characteristics of cells when scanned in forward and reverse directions (Supplementary Fig. 6). $1-\mathrm{cm}^{2}$ ultrathin solar cells are also fabricated on PEN substrates with AgNWs electrodes (with PBDB-T-2F:IT-4F active layer). The cell shows $V_{\mathrm{OC}}=0.85 \mathrm{~V}, J_{\mathrm{SC}}=19.6 \mathrm{~mA} \mathrm{~cm}^{-2}, \mathrm{FF}=0.67$ and $\mathrm{PCE}=11.2 \%$ which is comparable to the $1-\mathrm{cm}^{2}$ reference cell on glass substrate/ITO (Supplementary Fig. 7). The performance of the $1-\mathrm{cm}^{2}$ cells validates the effectiveness and reproducibility of the PEI-Zn interlayer for efficient electron collection.

Chemical compatibility and mechanical property of PEI-Zn. Development of PEI- $\mathrm{Zn}$ is the key part of the high-performance ultraflexible nonfullerene OSCs. To study the chemical compatibility of the PEI- $\mathrm{Zn}$ and the nonfullerene active layer, devices with a structure glass/ITO/PEI-Zn/PBDB-T-2F:IT-4F/MoO $3 / \mathrm{Ag}$ (Fig. 2a) were fabricated. Different content of $\mathrm{Zn}^{2+}$ was added to optimize the PEI-Zn interlayer. First, PEI (without $\mathrm{Zn}^{2+}$ ) is used as the interfacial layer in the nonfullerene OSCs. The cell shows poor performance with a pronounced " $\mathrm{S}$ " shape in the $J-V$ characteristics (Fig. 2b). Based on our previous study of the 
a
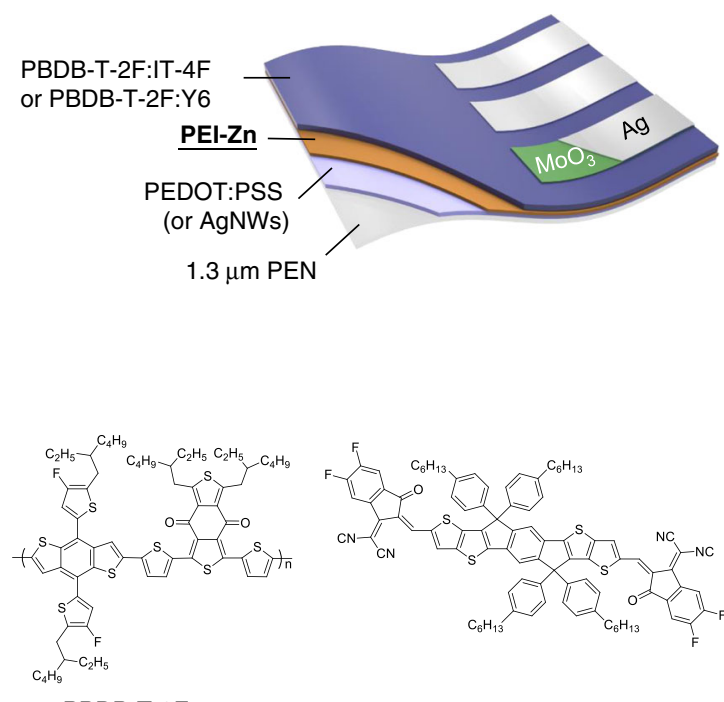

PBDB-T-2F

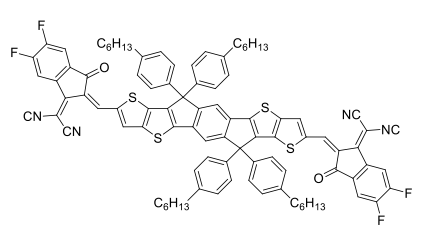

IT-4F b

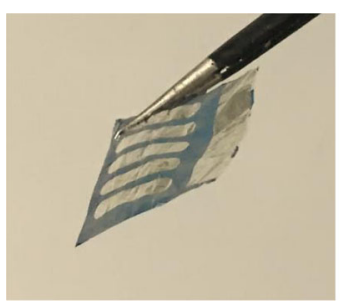

C

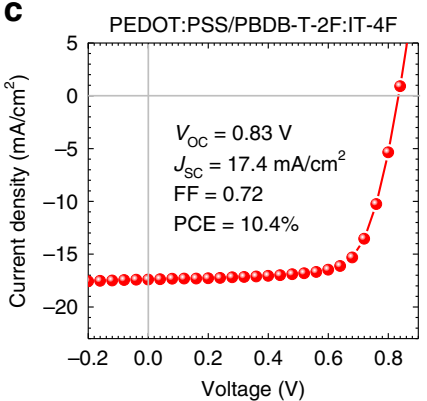

d

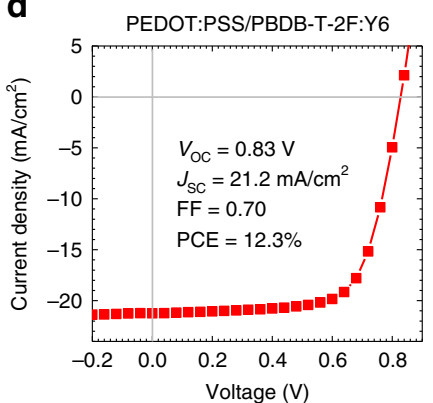

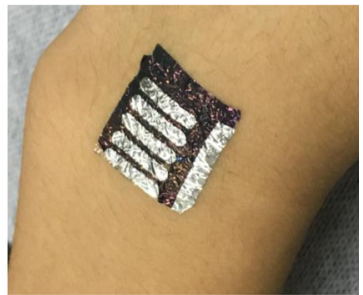

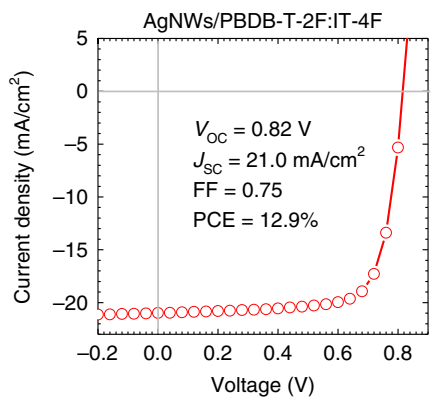

AgNWs/PBDB-T-2F:IT-4F

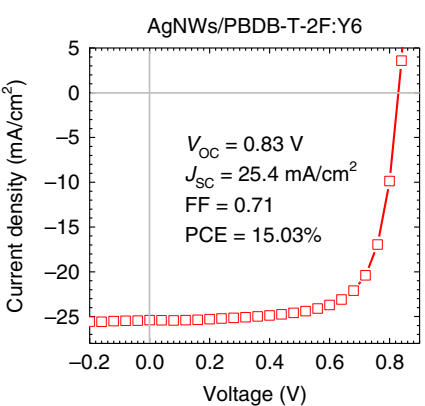

Fig. 1 Device structure and photovoltaic performance of the ultraflexible OSC. a Device structure of the ultrathin solar cells and chemical structure of the nonfullerene active layer; $\mathrm{A} \mathrm{Zn}^{2+}$-chelated polyethylenimine (PEI-Zn) is developed as the electron-transporting layer (ETL). $\mathbf{b}$ Pictures of the fabricated ultrathin OSCs; the picture on the right is a cell attached on the wrist. $\mathbf{c}, \mathbf{d} J-V$ characteristics of the ultrathin devices with conducting polymer (PEDOT:PSS) or $\mathrm{Ag}$ nanowires as the transparent electrodes. Two different nonfullerene active layers of PBDB-T-2F:IT-4F and PBDB-T-2F:Y6 were used in the ultraflexible OSCs.

reaction between PEIE and IEICO-4F nonfullerene acceptor, it can be inferred that the PEI can chemically react with the IT$4 \mathrm{~F}^{31}$. When PEI is added into the IT- $4 \mathrm{~F}$ chlorobenzene solution, the color of the IT-4F changed from dark blue to brown (Fig. 2c). Absorption band between 500 and $750 \mathrm{~nm}$ disappears (Fig. 2c). Combining ${ }^{1} \mathrm{H}$ Nuclear magnetic resonance (NMR) study (Supplementary Fig. 8) and the mass spectroscopy (Supplementary Fig. 9), it is suggested that the amine reacts with the $\mathrm{C}=\mathrm{C}$ linkage moiety through the Michael addition reaction that destroy the chemical and electronic structure of IT- $4 \mathrm{~F}^{35}$. The structure of PEI is complicated, and thus $1 \mathrm{H}$ NMR signals of the mixed solution of PEI and IT-4F are difficult to recognize. The absorption change of IT-4F solutions is similar when adding PEI and ethanolamine. So we used ethanolamine as the model compound to study the reaction. That leads to poor device performance. PEI was also added into the Y6 solution to study the reaction between Y6 and PEI. As shown in Supplementary Fig. 10a, color of Y6 solution changes from blue to light blue after adding PEI solution. Absorption band of Y6 between 600 and $800 \mathrm{~nm}$ becomes weaker. Meanwhile, solar cell with a structure of ITO/PEI/PBDB-T-2F: $\mathrm{Y} 6 / \mathrm{MoO}_{3} / \mathrm{Ag}$ displays a low FF of 0.45 and a poor PCE of $6.08 \%$ (Supplementary Fig. 10b).

To suppress the reaction between PEI and the nonfullerene acceptor IT-4F, $\mathrm{Zn}^{2+}$ is introduced to chelate with the PEI by adding zinc acetate into the PEI solution. $\mathrm{Zn}^{2+}$ is selected to chelate with the PEI because $\mathrm{Zn}^{2+}$ has strong chelation properties with nitrogen atoms (such as in the compounds of Zinc porphyrin $^{36}$, Zinc phthalocyanine ${ }^{37}$ ) and hydroxyl functional groups ${ }^{38}$. After the PEI is chelated with the $\mathrm{Zn}^{2+}$, the chemical reactivity of the PEI is reduced. As shown in Fig. $2 \mathrm{c}$, the IT- $4 \mathrm{~F}$ solution mixed with the $\mathrm{Zn}^{2+}$ chelated PEI shows similar color and absorption spectra to the pristine IT-4F solution. The similar phenomenon is observed when PEI-Zn is added into the Y6 solution (Supplementary Fig. 10a). Note that the Zn chelated $\mathrm{PEI}$ is different from the mixture of PEI and $\mathrm{ZnO}$ precursor which will react with IT-4F solution. The color of the IT-4F solution changes when the PEI: $\mathrm{ZnO}$ precursor is mixed with IT$4 \mathrm{~F}$ solution (Supplementary Fig. 11). For the solar cell performance, the ' $S$ ' shape in the $J-V$ characteristics gradually disappears as the content of $\mathrm{Zn}^{2+}$ increases (Fig. 2b). The photovoltaic parameters are summarized in Supplementary Table 1. The device with pure PEI displays a pronounced ' $\mathrm{S}$ ' shape with a low FF of 0.30 . When the $\mathrm{Zn}$-to-N mole ratio comes to $4: 1$, the "S" shape in $J-V$ curve disappears with a high performance $\left(V_{\mathrm{OC}}=0.82 \mathrm{~V}, J_{\mathrm{SC}}=20.7 \mathrm{~mA} \mathrm{~cm}^{-2}, \mathrm{FF}=0.73\right.$ and $\mathrm{PCE}=12.4 \%)$. The device performance is further improved with the mole ratio of $\mathrm{Zn}^{2+}: \mathrm{N}$ of $15: 1\left(V_{\mathrm{OC}}=0.84 \mathrm{~V}, J_{\mathrm{SC}}=20.8 \mathrm{~mA}\right.$ $\mathrm{cm}^{-2}, \mathrm{FF}=0.76$ and $\left.\mathrm{PCE}=13.3 \%\right)$. As the content of $\mathrm{Zn}$ $\left(\mathrm{CH}_{3} \mathrm{COO}\right)_{2}$ continues to increase, the mixed solution gets cloudy because the $\mathrm{Zn}\left(\mathrm{CH}_{3} \mathrm{COO}\right)_{2}$ cannot be fully coordinated with the PEI and dissolved. In addition to the zinc acetate, zinc acetylacetonate is also used to provide zinc source to coordinate 
a

\begin{tabular}{|c|}
\hline $\mathrm{MoO}_{3} / \mathrm{Ag}$ \\
\hline PBDB-T-2F:IT-4F \\
\hline PEI-Zn \\
\hline ITO \\
\hline Glass \\
\hline
\end{tabular}

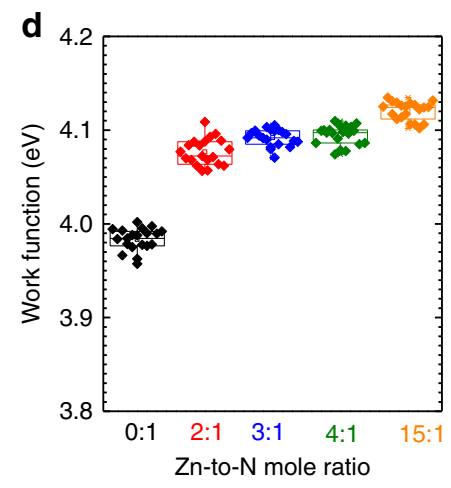

g

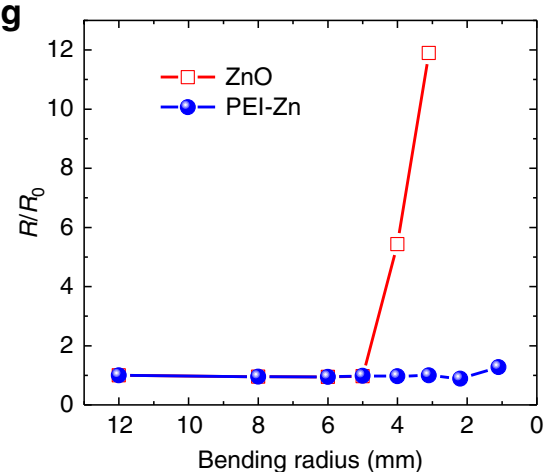

b

e
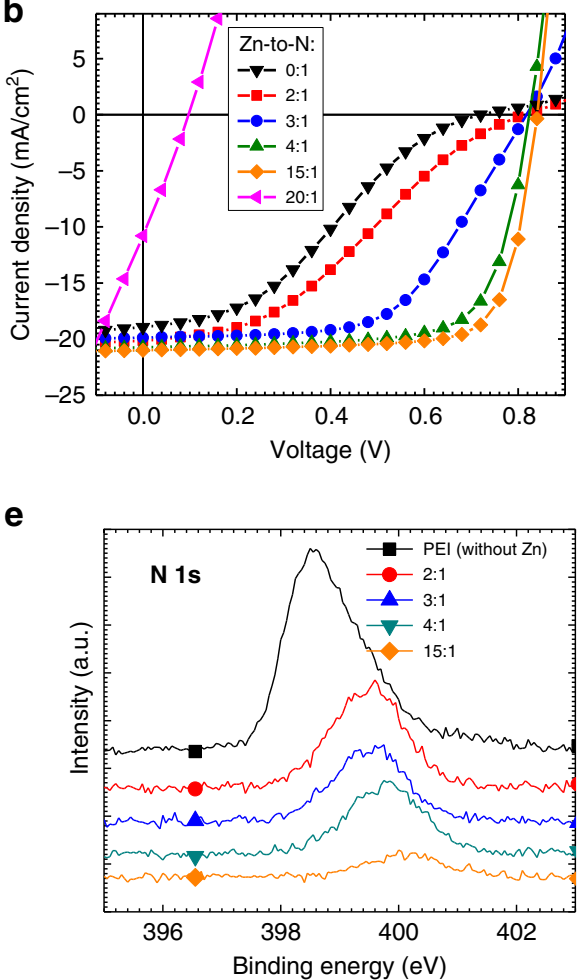

h

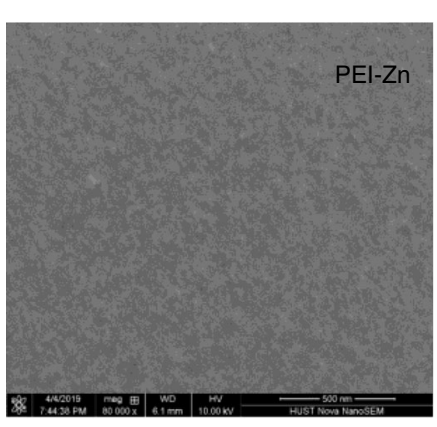

c

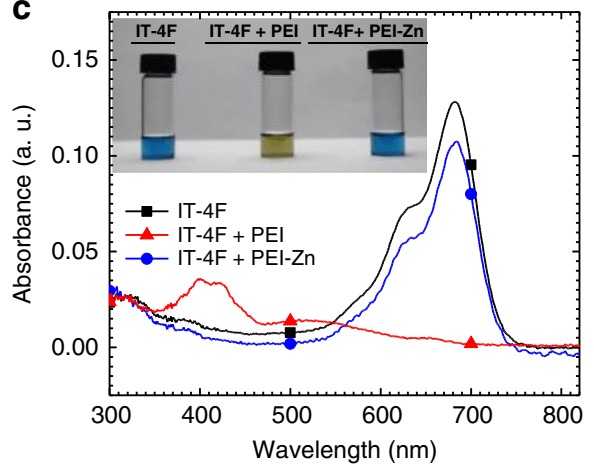

f

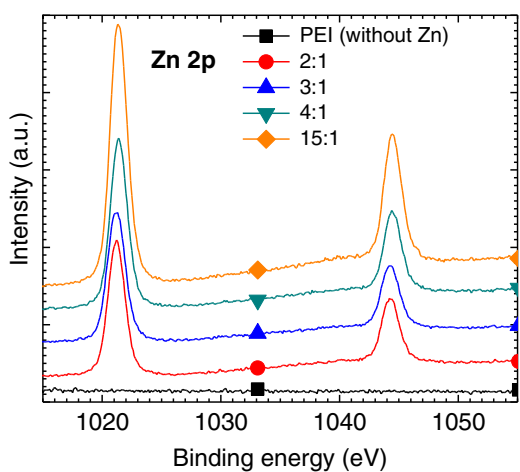

i

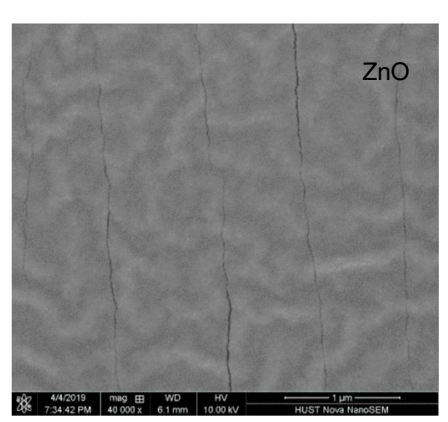

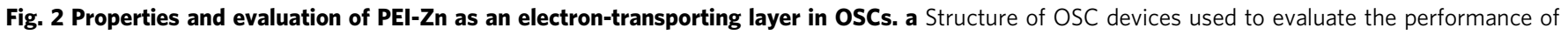
$\mathrm{PEI}-\mathrm{Zn}$ as an electron-transporting layer; $\mathbf{b} \mathrm{J}-\mathrm{V}$ characteristics of the devices with PEI-Zn ETL containing different $\mathrm{Zn}$-to- $\mathrm{N}$ mole ratios; $\mathrm{Zn}$-to- $\mathrm{N}$ mole ratio is shown in the legend. c Absorption spectra of three solutions: IT-4F, IT-4F mixed with PEI, and IT-4F mixed with PEI-Zn solution. The inset shows the

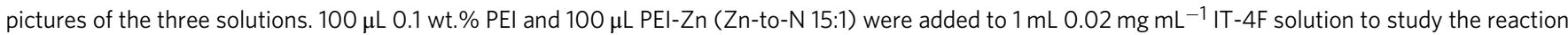
between them, respectively. d Work function of the PEI-Zn containing different Zn-to-N mole ratios; Boxes, square symbols, and horizontal bars indicate 25/75 percentile, mean, and $\mathrm{min} / \mathrm{max}$ values, respectively. X-ray photoelectron spectroscopy of the PEI-Zn films containing different Zn-to- $\mathrm{N}$ ratios: e $\mathrm{N}$ $1 \mathrm{~s} ; \mathbf{f} \mathrm{Zn} 2 \mathrm{p}$. $\mathbf{g}$ Strain test on the PEI-Zn and $\mathrm{ZnO}$ films deposited on $175-\mu \mathrm{m}$ thick PES substrates. Resistance ratio of the films after bending ( $R$ ) over the

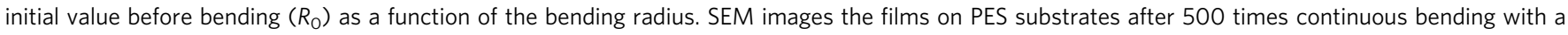
bending radius of $4 \mathrm{~mm}$ : $\mathbf{h} \mathrm{PEI}-\mathrm{Zn}$ film; i $\mathrm{ZnO}$ film.

with the PEI. Devices based on PEI-Zn with different ratios of zinc acetylacetonate are also evaluated. The performance shows a similar trend to the case of zinc acetate used as the zinc source (Supplementary Fig. 12). The photovoltaic performance is summarized in Supplementary Table 2.

Different thicknesses of PEI-Zn ETL were tested in solar cells with PBDB-T-2F:IT-4F active layer. The devices show comparable performance in the range of $12.3-12.8 \%$ when the thickness of PEI-Zn changes from 30-80 nm (Supplementary Fig. 13). Photovoltaic data are summarized in Supplementary Table 3. Electrical conductivity of the PEI-Zn (Zn-to-N, 15:1) was measured using a transmission line method to exclude the influence of contact resistance with a structure in Supplementary
Fig. 14a. The electrical conductivity of PEI-Zn (Zn-to-N, 15:1) is $3.4 \times 10^{-6} \mathrm{~S} \mathrm{~cm}^{-1}$. Preparation of PEI-Zn includes two stages: (1) Zinc ion is added into the PEI alcohol solution. Based on the suppressed reaction between PEI-Zn solution and IT-4F (Fig. 1c), it can be inferred that the electron lone pairs on the nitrogen interacts with zinc ion; (2) A thermal annealing $\left(110-150{ }^{\circ} \mathrm{C}\right)$ treatment is applied on the PEI-Zn. From the absorption and photoluminescence spectra of the film (Supplementary Fig. 15a, b), there is possible formation of $\mathrm{ZnO}$ in the film after the thermal annealing. The long backbone of PEI (molecular weight: 25000) along with the $\mathrm{ZnO}$ could be the reason of the film simultaneously displaying good mechanical robustness and a conductivity of $3.4 \times 10^{-6} \mathrm{~S} \mathrm{~cm}^{-1}$. 
Figure $2 \mathrm{~d}$ shows the work function of PEI-Zn containing different content of $\mathrm{Zn}^{2+}$ measured by Kelvin probe. The reference sample of PEI coated on ITO is about $4.0 \mathrm{eV}$. The value slightly increases to $4.1 \mathrm{eV}$ after adding $\mathrm{Zn}^{2+}$, and the work function is almost independent on the content of $\mathrm{Zn}^{2+}$. The PEI$\mathrm{Zn}$ remains the advantage of PEI that can produce low work function and is beneficial to the electron collection in OSCs. Xray photoelectron spectroscopy (XPS) measurement was performed to confirm the chelation between PEI and $\mathrm{Zn}^{2+}$. As shown in Fig. 2e, the binding energy of N $1 \mathrm{~s}$ is $398.55 \mathrm{eV}$ for neat PEI. When the $\mathrm{Zn}^{2+}$ ions are introduced, the binding energy of $\mathrm{N}$ $1 \mathrm{~s}$ shifts to blue. As the $\mathrm{Zn}^{2+}$ content increases, more $\mathrm{N}$ (electron lone pairs) chelated with $\mathrm{Zn}^{2+}$. Thus, the shift of binding energy gets larger. The binding energy of $\mathrm{Zn} 2 \mathrm{p}$ also shifts to blue as the content of $\mathrm{Zn}^{2+}$ increases (Fig. 2f). These results indicate that electron transfer from $\mathrm{N}$ of PEI to $\mathrm{Zn}^{2+}$ for the coordination. This electron transfer weakens the electronegativity of amine in PEI, which suppresses the reaction of PEI and IT-4F and improves the solar cell performance. Besides $\mathrm{Zn}^{2+}$, we have also tried other metal ions, including $\mathrm{Cu}^{2+}, \mathrm{Ni}^{2+}, \mathrm{Mn}^{2+}$ and $\mathrm{Ag}^{+}$. They can also chelate with amine ${ }^{39}$. However, their work function values $(4.60 \mathrm{eV}$ for PEI-Cu, $4.42 \mathrm{eV}$ for PEI-Ni, $4.68 \mathrm{eV}$ for PEI$\mathrm{Mn}$, and $4.32 \mathrm{eV}$ for PEI-Ag, measured by Kelvin Probe) are not as low as that of PEI-Zn $(4.1 \mathrm{eV})$ for efficient electron collection.

Figure $2 \mathrm{~g}$ shows the critical strain test on the PEI- $\mathrm{Zn}$ and $\mathrm{ZnO}$ reference films. The films were deposited on $175-\mu \mathrm{m}$ thick polyethersulfone (PES) substrates. The individual films on PES were bended at different bending radius and their resistance was measured before and after bending. For $\mathrm{ZnO}$ film, the resistance starts to increase when the bending radius $(r)$ is less than $5 \mathrm{~mm}$. For PEI-Zn film, the resistance keeps unchanged until $r$ is as low as $2.2 \mathrm{~mm}$. According to the bending strain equation $\left(\varepsilon=H_{\mathrm{s}} /(2 r)\right.$, where $H_{s}$ is the thickness of the PES substrate), the maximum bending strain of PEI-Zn (3.98\%) is over twice as high as that of $\mathrm{ZnO}(1.75 \%)$. Figure $2 \mathrm{~h}, \mathrm{i}$ are the scanning electron microscope (SEM) images of PEI-Zn and $\mathrm{ZnO}$ films on PES substrates after bending for 500 times with a bending radius of $4 \mathrm{~mm}$. No cracks were observed on the SEM image of PEI-Zn film after the continuous bending (Fig. 2h). On the contrary, cracks can be observed for $\mathrm{ZnO}$ film after the same bending conditions (Fig. 2i).

Mechanical robustness of the ultraflexible OSCs. To test mechanical robustness of the ultraflexible cell, we transferred the cell to a pre-strained elastomer, VHB, with $300 \%$ of the initial length. The cell is at flat or compressed state by stretching and releasing the elastomer, as shown in Fig. 3a. When the elastomer is at the pre-strained state, the ultrathin cell is flat. When the elastomer is released, the cell is at the compressed state. The cycling was performed on the apparatus shown in Supplementary Fig 16. At the compressed state, wrinkled stripes can be observed perpendicular to the direction of strained force. The radius of the wrinkles reaches lower than $20 \mu \mathrm{m}$ (Fig. 3b). When the elastomer is stretched, the wrinkles become sparser and finally disappear at the flat state (Supplementary Fig. 17). The deformation is quantified as $\Delta L / L_{0}$, where $L_{0}$ is the length of the cell when the cell is at flat state (defined as the initial state), and $\Delta L$ is the changed length of the cell when elastomer is released. Supplementary Fig. 18 shows the current-voltage $(I-V)$ characteristics of the cell at different deformation states. The $V_{\mathrm{OC}}$ and $\mathrm{FF}$ remain high almost without change $\left(V_{\mathrm{OC}}=0.83 \mathrm{~V}\right.$ and $\left.\mathrm{FF}=0.70\right)$ when the cell is at compression ratio up to $45 \%\left(\Delta L / L_{0}\right)$.

To further test the mechanical robustness of the cells, continuous deformation cycling test (45\% compression and flat) has been performed. Cells with different electrodes (ITO, AgNWs and PEDOT:PSS) and different ETL (PEI-Zn and $\mathrm{ZnO}$ ) have been fabricated. Ultraflexible OSCs with PEI-Zn show higher PCE for all tested different structures, compared to $\mathrm{ZnO}$ (Supplementary Fig. 19). Figure $3 \mathrm{c}$ shows the ultrathin cells with ITO electrodes degrade very rapidly during the cycling test. The performance is reduced to about $10 \%$ of its initial PCE after one-time cycling. When ZnO ETL is replaced by PEI-Zn, the cell shows improved mechanical property. The PCE remains 60 and $40 \%$ of initial PCE after one-time and seven times cycling. But, the overall mechanical robustness is still poor, which is due to the brittle properties of ITO electrode ${ }^{40}$. It should be note that there is no barrier layer here (such as parylene) coated on top of the ITObased ultrathin cells. The barrier layer enables the ITO embedding at a neutral strain position and suffering less strain, and therefore better mechanical robustness was achieved in the previous work ${ }^{21,25}$. The ITO here suffers higher stain and the test condition is harsher. Figure $3 \mathrm{~d}$ shows the mechanical properties of the cells with AgNWs electrodes. The PEI-Zn ETL-based cells also deliver better mechanical robustness than the ZnO ETLbased cell. After the first cycle, the efficiency becomes $77 \%$ of initial PCE for the ZnO ETL-based cell, while 97\% of its initial PCE for the PEI-Zn ETL-based cell. After 100 deformation cycling, the PEI-Zn ETL-based cell still has $89 \%$ of its initial PCE. When the transparent electrode is changed to PEDOT:PSS, PCE of the cell (with PEI-Zn ETL) is almost unchanged during the 100 continuous deformation cycles (Fig. 3e). These results suggest the PEI-Zn can deliver good mechanical properties for the ultraflexible solar cells even not at a neutral strain position. Note that mechanical flexibility of cells with PEDOT:PSS/ZnO is not studied for comparison because the device showed poor performance, which is associated the chemical reduction of PEDOT:PSS by sol-gel ZnO. The color of PEDOT:PSS film changed from light blue to dark blue after spin-coating $\mathrm{ZnO}$ with thermal annealing (Supplementary Fig. 20). Two peaks at around $650 \mathrm{~nm}$ and $950 \mathrm{~nm}$ are observed in the absorption spectrum of PEDOT:PSS/ZnO, which are assigned to the polaron-induced absorption and neutralized absorption of PEDOT ${ }^{41}$. Sheet resistance of PEDOT:PSS film increases from $110 \mathrm{ohm} \mathrm{sq} \mathrm{s}^{-1}$ to around $430 \mathrm{ohm} \mathrm{sq}^{-1}$ after the $\mathrm{ZnO}$ coating. The increased absorbance and sheet resistance result in poor performance.

Then, we attached a cell on a finger joint to demonstrate its functioning under compressing-releasing cycles. Under a solar simulator, the current of the stretchable solar cells changes periodically with the finger bending and spreading. Supplementary Movies 1 and 2 show the compressing-releasing cycling of the ultrathin cell on the stretching-releasing apparatus and the bending-spreading finger, separately. The current of the cell under the solar simulator could change back and forth during the cycling recorded by the multimeter. Fig. $3 \mathrm{f}$, g show the pictures captured from the supporting videos that show the current recovers during the cycling.

\section{Discussion}

We have presented a low-work function interlayer of $\mathrm{Zn}^{2+}$ chelated polyethylenimine (PEI-Zn) that can endure high mechanical bending strain, and simultaneously has good chemical compatibility with the efficient nonfullerene active layers. Using the PEI-Zn interlayer, ultraflexible solar cells with PCE up to $15 \%$ have been demonstrated. Furthermore, the ultraflexible solar cells show nearly unchanged PCE during 100 continuous compression-flat deformation cycles with a compression ratio of $45 \%$. The PEI-Zn layer is solution-processed and works efficiently with the printable flexible transparent electrodes of metal nanowires (such as AgNWs) and conducting polymer PEDOT:PSS. Cells with doctor-bladed AgNWs/PEI-Zn electrodes show comparable performance to spin-coated electrodes (Supplementary 
a

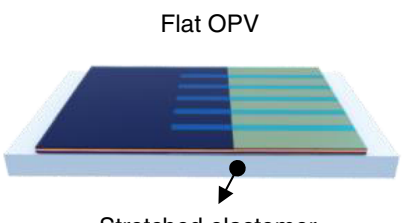

Stretched elastomer

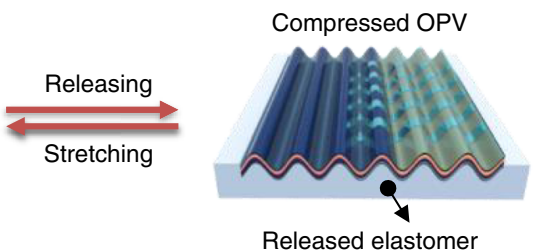

Released elastomer

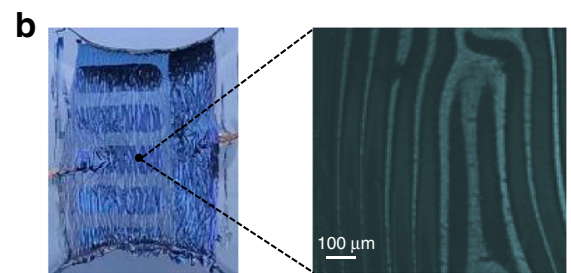

d

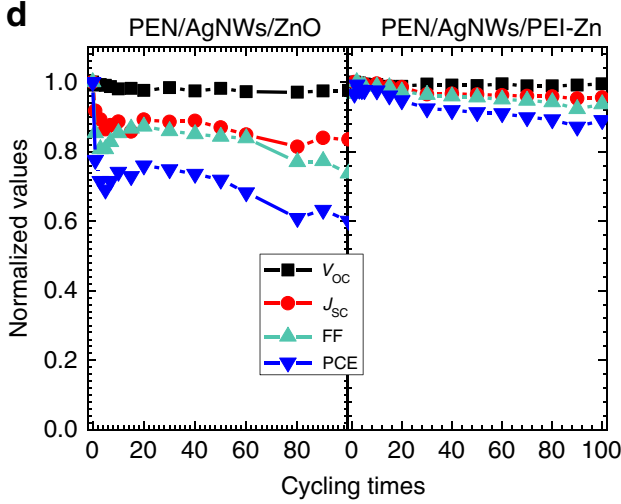

\section{e}

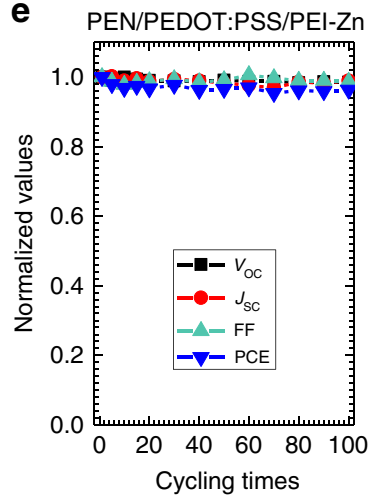

C

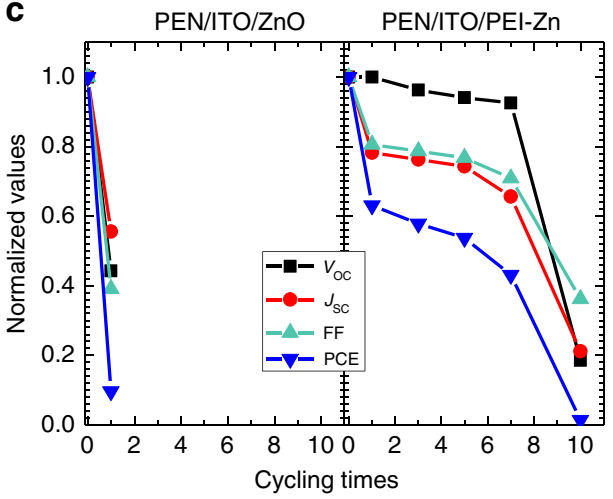

f

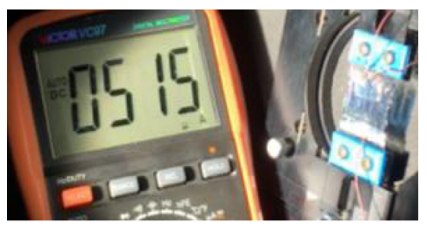

g

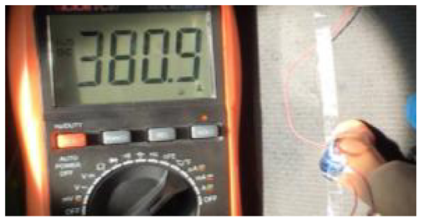

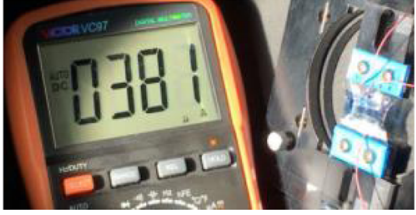
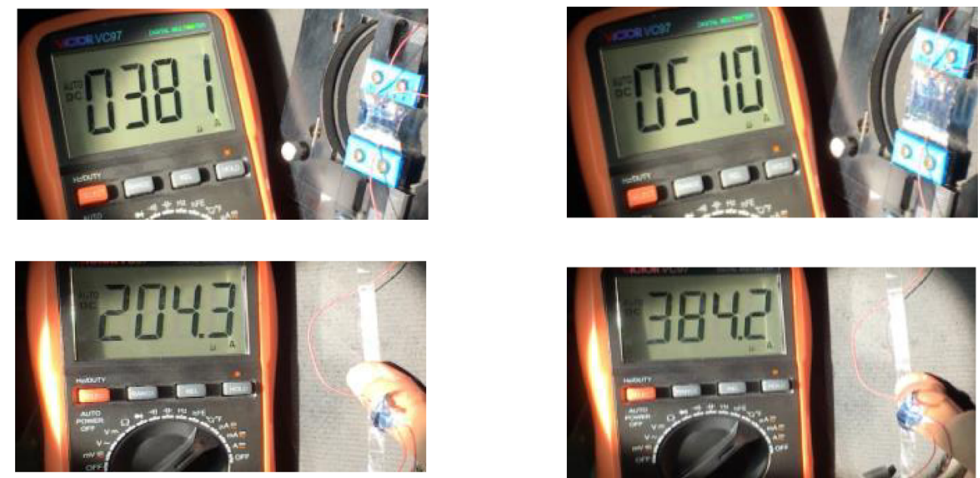

Fig. 3 Extreme mechanical flexibility. a Illustration of the ultrathin OSCS attached onto the 300\% pre-stretched elastomer (VHB) to demonstrate the extreme flexibility; the cells are at flat and compressed states by stretching and releasing the elastomer. When the elastomer is stretched to the prestretched state, the ultrathin cell becomes flat. When the elastomer is released, the cell is at the compressed state. $\mathbf{b}$ Picture of ultrathin cells on a released elastomer with a $45 \%$ compression. The right is an image of an compressed cell under an optical microscope; Evolution of photovoltaic parameters after different compressed-flat cycles of ultraflexible of cells with different electrode/ETL: c with PEN/ITO/ZnO and PEN/ITO/PEI-Zn; d with PEN/AgNWs/ $\mathrm{ZnO}$ and PEN/AgNWs/PEI-Zn; e with PEN/PEDOT:PSS/PEI-Zn; Pictures captured from the supplementary videos to demonstrate the current recovery during the cycling: $\mathbf{f}$ cell on a stretching-releasing apparatus; $\mathbf{g}$ cell attached on a finger joint.

Fig. 21). The PEI-Zn can be very promising in printed electronics and flexible electronics.

\section{Methods}

Materials. PBDB-T-2F and IT-4F were purchased from Solarmer Materials Inc. Y6 is purchased eFlexPV Technology (Shenzhen) Co., Ltd. Zinc acetate dihydrate, methoxyethanol, PEIE (80\% ethoxylated solution, 37 wt.\% in $\mathrm{H}_{2} \mathrm{O}$ ) and polyethylenimine (PEI, Mw 25000) for PEI-Zn precursor solutions were purchased from Sigma-Aldrich. PEDOT:PSS aqueous solution (Clevios PH1000) was purchased from Xi'an Polymer Light Technology Corp. Ethylene glycol, and 1.3- $\mu$ m-thick PEN films were purchased from Sigma-Aldrich. The sol-gel $\mathrm{ZnO}$ solution was prepared by dissolving $0.1 \mathrm{~g}$ Zinc acetate dihydrate and $0.028 \mathrm{~g}$ ethanolamine (Sigma-Aldrich) in $1 \mathrm{~mL}$ methoxyethanol. To prepare PEI-Zn precursors, $0.1 \mathrm{wt}$ \% PEI or $0.5 \mathrm{wt} . \%$ PEIE (plus water) was dissolved in methoxyethanol. Then, different amount of zinc acetate dihydrate or zinc acetylacetonate was added into the PEI or PEIE solution, and stirred for $3 \mathrm{~h}$ before coating. For the optimized $\mathrm{Zn}$-to-N mole ratio of 15:1, $0.075 \mathrm{~g}$ zinc acetate dihydrate was added to the $1 \mathrm{~mL} 0.1 \mathrm{wt} . \%$ PEI or $0.5 \mathrm{wt} . \%$ PEIE (plus water) solution and stirred until the solution became clear. Adding a small amout $(10 \mu \mathrm{L})$ of water faciliates the dissolving of Zinc acetate dihydate or Zinc acetylacetonate hydrate in PEI or PEIE solution.

Device fabrication. The ultrathin PEN substrates were firstly cut into the size of around $2.3 \times 2.3 \mathrm{~cm}^{2}$ and adhered onto PDMS-coated glass substrates. PDMS films was prepared by a mixture of base and crosslinker (10:1, weight ratio, PP2-OE41, Gelest Inc.) and cured on a hot plate in air at $80^{\circ} \mathrm{C}$ for $40 \mathrm{~min}$. The bottom electrode of PEDOT:PSS was prepared by spin-coating the PH1000 consisting of 5 wt.\% ethylene glycol and 0.1 wt.\% superwet-340 surfactant and patterned through surface energy tuning via plasma treatment. As for AgNWs electrode, AgNWs solution of $2 \mathrm{mg} \mathrm{mL}^{-1}$ was spin-coated on substrates at a speed of $2000 \mathrm{rpm}$, following an annealing at $200^{\circ} \mathrm{C}$ for $10 \mathrm{~min}$. PEDOT:PSS and AgNWs electrodes were patterned under a fetosecond laser (Hongtuo, Wuhan). Next, PEI-Zn precursor solution was spin-coated at $3000 \mathrm{rpm}$ for $40 \mathrm{~s}$, following a thermal annealing at $150{ }^{\circ} \mathrm{C}$ for $10 \mathrm{~min}$. The PEI-Zn was spin-coated on PEDOT:PSS electrodes and AgNWs electrodes (PEI-Zn was spin-coated twice on AgNWs to flatten the surface). When the mole ratio of $\mathrm{Zn}$-to- $\mathrm{N}$ was smaller than $3: 1$, the films were thinner than $10 \mathrm{~nm}$. The thicknesses were 12,19 , and $25 \mathrm{~nm}$ for the films prepared from precursors with mole ratios (Zn-to-N) of 4:1, 10:1 and 15:1, respectively. Then, the substrates were transferred to the $\mathrm{N}_{2}$-filled glovebox. PBDB-T-2F:IT-4F (1:1, weight ratio) in a $20 \mathrm{mg} \mathrm{mL}^{-1}$ chlorobenzene:DIO (99.5:0.5, volume ratio) solution was spin-coated at $1500 \mathrm{rpm}$ for $60 \mathrm{~s}$. PBDB-T-2F:Y6 (1:1.2, weight ratio) in a $16 \mathrm{mg} \mathrm{mL}^{-1}$ chloroform:chloronaphthalene (CN) (99.5:0.5, volume ratio) solution was spin-coated at $2500 \mathrm{rpm}$ for $60 \mathrm{~s}$. After a thermal annealing at $100{ }^{\circ} \mathrm{C}$ for $10 \mathrm{~min}$, the samples were transferred into the vacuum evaporation system (Minispectros, Kurt J. Lesker). 7-nm-thick $\mathrm{MoO}_{3}$ and 100-nm-thick Ag were evaporated with a metal shadow mask under high vacuum $\left(<5 \times 10^{-7}\right.$ Torr $)$. The area of single cell is $4.1 \mathrm{~mm}^{2}$. Before measurement, the devices were annealed at $100{ }^{\circ} \mathrm{C}$ for $10 \mathrm{~min}$ in the $\mathrm{N}_{2}$-filled glove box. Stretching-releasing apparatus and operational procedure were shown in Supplementary Fig. 16 and Supplementary Movie 1. 
Characterization of film and devices. Current density-voltage $(J-V)$ characteristics of the cells were measured using a Keithley 2400 SourceMeter. The cells were illuminated through an aperture area of $4.095 \mathrm{~mm}^{2}$ from a $100 \mathrm{~mW} \mathrm{~cm}{ }^{-2}$ AM1.5 solar simulator (Newport, ORIEL, Sol3A, $450 \mathrm{~W}$ xenon lamp). The longterm photostability of devices was tested with an ultraviolet filter the cut off the light illumination below $400 \mathrm{~nm}$. The area of the aperture is confirmed by the National Institute of Metrology (NIM, Beijing). The measurement was performed in a $\mathrm{N}_{2}$-fillerd glove box at the temperature of $25^{\circ} \mathrm{C}$. The light source is a solar simulator (Newport, Sol3A) with a 450 watt xenon lamp (Newport). Light intensity of the light source was calibrated using a NIST-certified monocrystalline Si solar cell (Newport 532 ISO1599). There is almost no variation of the performance with or without aperture. The cells were measured with a step voltage of $0.04 \mathrm{~V}$ and a dwell time of $0.2 \mathrm{~s}$ for every point. Absorbance and transmittance of films was characterized using a UV-vis-NIR Spectrophotometer (UV3600, Shimadzu). Reflectance of the cells with different deformation ratios was also conducted by the UV-vis-NIR Spectrophotometer with an integrating sphere. The valence states of $\mathrm{Zn}$ and $\mathrm{N}$ were measured by X-ray photoelectron spectrometer (XPS) (Kratos Analytical). The SEM images of PEI-Zn (Zn-to-N ratio of 15:1) and ZnO after bending for 500 times were conducted by FEI Nova Nano-SEM 450. The work function was carried out by Kelvin probe (KP-020, KP Technology). The photographic images of the ultrathin devices on a pre-strained elastomeric tape under different compression were taken by using optical microscope (Nikon ECLIPSE LV150). The EQE test was performed by a standard system using a $150 \mathrm{~W}$ xenon lamp (Newport) fitted with a monochromator (Cornerstone 74004) as a monochromatic light source. The NMR spectra were collected on a Bruker Ascend 400 MHZ NMR spectrometer using deuterated dichloromethane as the solvent and tetramethylsilane as the internal standard. The liquid chromatography-mass spectrometry measurement was performed on an Agilent 1100 LC-MS Trap XCT System (Agilent Technologies, USA).

Reporting summary. Further information on research design is available in the Nature Research Reporting Summary linked to this article.

\section{Data availability}

The data that support the findings of this study are available from the corresponding author upon reasonable request. Source data are provided with this paper.

Received: 28 April 2020; Accepted: 10 August 2020;

Published online: 09 September 2020

\section{References}

1. Hou, J. et al. Organic solar cells based on non-fullerene acceptors. Nat. Mater. 17, 119-128 (2018)

2. Kolesov, V. A. et al. Solution-based electrical doping of semiconducting polymer films over a limited depth. Nat. Mater. 16, 474-480 (2017).

3. Meng, L. et al. Organic and solution-processed tandem solar cells with $17.3 \%$ efficiency. Science 361, 1094-1098 (2018).

4. Cheng, P. et al. Next-generation organic photovoltaics based on non-fullerene acceptors. Nat. Photonics 12, 131-142 (2018).

5. Zhao, J. et al. Efficient organic solar cells processed from hydrocarbon solvents. Nat. Energy 1, 15027 (2016).

6. Yan, C. et al. Non-fullerene acceptors for organic solar cells. Nat. Rev. Mater. 3, 18003 (2018).

7. Qian, D. et al. Design rules for minimizing voltage losses in high-efficiency organic solar cells. Nat. Mater. 17, 703-709 (2018).

8. Zhang, J. et al. Material insights and challenges for non-fullerene organic solar cells based on small molecular acceptors. Nat. Energy 3, 720-731 (2018).

9. Du, X. et al. Efficient polymer solar cells based on non-fullerene acceptors with potential device lifetime approaching 10 years. Joule 3, 215-226 (2019).

10. Meng, W. et al. Conductivity enhancement of PEDOT:PSS films via phosphoric acid treatment for flexible all-plastic solar cells. ACS Appl. Mater. Interfaces 7, 14089-14094 (2015).

11. Sun, L. et al. Flexible All-Solution-Processed Organic Solar Cells with HighPerformance Nonfullerene Active Layers. Adv. Mater. 32, 1907840 (2020).

12. Kaltenbrunner, M. et al. An ultra-lightweight design for imperceptible plastic electronics. Nature 499, 458-463 (2013).

13. $\mathrm{Xu}$, J. et al. Highly stretchable polymer semiconductor films through the nanoconfinement effect. Science 355, 59-64 (2017).

14. Lipomi, D. J. Organic photovoltaics: focus on its strengths. Joule 2, 195-198 (2018).

15. Someya, T. et al. The rise of plastic bioelectronics. Nature 540, 379-385 (2016).

16. Bauer, $\mathrm{S}$. et al. 25th anniversary article: a soft future: from robots and sensor skin to energy harvesters. Adv. Mater. 26, 149-161 (2014).
17. Yokota, T. et al. Ultraflexible organic photonic skin. Sci. Adv. 2, e1501856 (2016).

18. Tee, B. C.-K. et al. A skin-inspired organic digital mechanoreceptor. Science 350, 313-316 (2015).

19. Wu, W. et al. Materials and wearable devices for autonomous monitoring of physiological markers. Adv. Mater. 30, 1705024 (2018).

20. Su, Y. et al. In-plane deformation mechanics for highly stretchable electronics. Adv. Mater. 29, 1604989 (2017).

21. Park, S. et al. Self-powered ultra-flexible electronics via nano-gratingpatterned organic photovoltaics. Nature 561, 516-521 (2018).

22. Kimura, H. et al. High operation stability of ultraflexible organic solar cells with ultraviolet-filtering substrates. Adv. Mater. 31, 1808033 (2019).

23. Lipomi, D. J. et al. Stretchable organic solar cells. Adv. Mater. 23, 1771-1775 (2011).

24. Kaltenbrunner, M. et al. Ultrathin and lightweight organic solar cells with high flexibility. Nat. Commun. 3, 770 (2012).

25. Jinno, H. et al. Stretchable and waterproof elastomer-coated organic photovoltaics for washable electronic textile applications. Nat. Energy 2, $780-785$ (2017).

26. $\mathrm{Xu}$, X. et al. Thermally stable, highly efficient, ultraflexible organic photovoltaics. Proc. Natl Acad. Sci. USA. 115, 4589-4594 (2018).

27. Jiang, Z. et al. Durable ultraflexible organic photovoltaics with novel metaloxide-free cathode. Adv. Funct. Mater. 29, 1808378 (2018).

28. Song, W. et al. All-solution-processed metal-oxide-free flexible organic solar cells with over $10 \%$ efficiency. Adv. Mater. 30, 1800075 (2018).

29. Zhou, Y. et al. A universal method to produce low-work function electrodes for organic electronics. Science 336, 327-332 (2012).

30. Hu, L. et al. Chemical reaction between an ITIC electron acceptor and an amine-containing interfacial layer in non-fullerene solar cells. J. Mater. Chem. A 6, 2273-2278 (2018).

31. Xiong, S. et al. $12.5 \%$ flexible nonfullerene solar cells by passivating the chemical interaction between the active layer and polymer interfacial layer. Adv. Mater. 31, 1806616 (2019).

32. Zheng, Z. et al. A highly efficient non-fullerene organic solar cell with a fill factor over 0.80 enabled by a fine-tuned hole-transporting layer. Adv. Mater. 30, 1801801 (2018).

33. Zhao, W. et al. Molecular optimization enables over $13 \%$ efficiency in organic solar cells. J. Am. Chem. Soc. 139, 7148-7151 (2017).

34. Yuan, J. et al. Single-junction organic solar cell with over 15\% efficiency using fused-ring acceptor with electron-deficient core. Joule 3, 1140-1151 (2019).

35. Li, Z. et al. Highly sensitive built-in strain sensors for polymer composites: fluorescence turn-on response through mechanochemical activation. Adv. Mater. 28, 6592-6597 (2016).

36. Yamamoto, T. et al. Synthesis and chemical properties of $\pi$-conjugated zinc porphyrin polymers with arylene and aryleneethynylene groups between zinc porphyrin units. Macromolecules 33, 5988-5994 (2000).

37. Khoza, P. et al. Synthesis and photophysicochemical properties of zinc phthalocyanine derivatized with benzothiazole or carbazole photosensitizers. Polyhedron 61, 119-125 (2013).

38. Wen, X. et al. Tetrahydroxy-perylene bisimide embedded in a zinc oxide thin film as an electron-transporting layer for high-performance non-fullerene organic solar cells. Angew. Chem. Int. Ed. 58, 13051-13055 (2019).

39. Moeller, T. et al. The coordination chemistry of yttrium and the rare earth metal ions. Chem. Rev. 65, 1-50 (1964).

40. Cairns, D. R. et al. Strain-dependent electrical resistance of tin-doped indium oxide on polymer substrates. Appl. Phys. Lett. 76, 1425-1427 (2000).

41. Liu, T. et al. Reduction and oxidation of poly(3,4-ethylenedioxythiophene): poly(styrenesulfonate) induced by methylamine (CH3NH2)-containing atmosphere for perovskite solar cells. J. Mater. Chem. A 4, 4305-4311 (2016).

\section{Acknowledgements}

The work is supported by the National Natural Science Foundation of China (Grant No. 51773072, 21474035), the HUST Innovation Research Fund (Grant No. 2016JCTD111, 2017KFKJXX012), the Science and Technology Program of Hubei Province (2017AHB040) and China Postdoctoral Science Foundation funded project (2016M602289). We thank Professor Jianfeng Zang for providing the stretching-releasing apparatus. We also thank the Analytical and Testing Center of Huazhong University of Science and Technology for providing facilities for characterization.

\section{Author contributions}

F.Q. and Y.H.Z. conceived the idea. F.Q. and W.W. performed the fabrication, measurement and optimization of ultrathin organic solar cells on PEDOT:PSS electrodes. F.Q. and L.L.S. performed the fabrication, measurement and optimization of ultrathin organic solar cells on AgNWs electrodes. L.H., S.X.X., T.F.L., X.S.J., and Y.Y.J. performed measurement of the conductivity, XPS, work function of PEI-Zn. F.Q., X.Y.D., and J.L. performed the compressing-releasing cycling measurement of the ultrathin solar cells. 
K.F. fabricated the indium tin oxide electrode on ultrathin PEN substrates and analyzed the results. Y.H.Z., J.H.H., and T.S. coordinated the work. F.Q. wrote the first draft of the manuscript. All the authors revised and approved the manuscript.

\section{Competing interests}

The authors declare no competing interests.

\section{Additional information}

Supplementary information is available for this paper at https://doi.org/10.1038/s41467020-18373-0.

Correspondence and requests for materials should be addressed to Y.Z.

Peer review information Nature Communications thanks Ning Li and the other, anonymous, reviewer(s) for their contribution to the peer review of this work.

Reprints and permission information is available at http://www.nature.com/reprints
Publisher's note Springer Nature remains neutral with regard to jurisdictional claims in published maps and institutional affiliations.

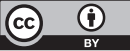

Open Access This article is licensed under a Creative Commons Attribution 4.0 International License, which permits use, sharing, adaptation, distribution and reproduction in any medium or format, as long as you give appropriate credit to the original author(s) and the source, provide a link to the Creative Commons license, and indicate if changes were made. The images or other third party material in this article are included in the article's Creative Commons license, unless indicated otherwise in a credit line to the material. If material is not included in the article's Creative Commons license and your intended use is not permitted by statutory regulation or exceeds the permitted use, you will need to obtain permission directly from the copyright holder. To view a copy of this license, visit http://creativecommons.org/ licenses/by/4.0/.

(C) The Author(s) 2020 